\title{
Healthspan Improvements in Caenorhabditis elegans with Traditional Chinese Herbal Tea
}

\author{
Chunxiu Lin $\mathbb{D}^{1,2}$ Xiaoying Zhang, ${ }^{1,2}$ Chuting Zhuang, ${ }^{1,2}$ Yugui Lin $\mathbb{D}^{3}{ }^{3}$ Yong Cao $\mathbb{D},{ }^{1,2}$ \\ and Yunjiao Chen $\left(^{1,2}\right.$ \\ ${ }^{1}$ Guangdong Provincial Key Laboratory of Nutraceuticals and Functional Foods, College of Food Science, South China \\ Agricultural University, Guangzhou, Guangdong, China \\ ${ }^{2}$ Guangdong Laboratory for Lingnan Modern Agriculture, Guangzhou, 510642 Guangdong, China \\ ${ }^{3}$ Department of Microbiology, Guangxi Medical University, Nanning, China
}

Correspondence should be addressed to Yong Cao; caoyong2181@126.com and Yunjiao Chen; yunjiaochen@scau.edu.cn

Received 27 January 2020; Revised 15 November 2020; Accepted 29 November 2020; Published 16 December 2020

Academic Editor: Felipe L. de Oliveira

Copyright (C) 2020 Chunxiu Lin et al. This is an open access article distributed under the Creative Commons Attribution License, which permits unrestricted use, distribution, and reproduction in any medium, provided the original work is properly cited.

Searching for natural and safe herbal tea with health benefits has attracted more and more attention, which is of great significance for reducing disease risk. A Chinese traditional herbal tea (HT) is rich in active ingredients extracted from natural plants. Numerous pharmacological studies showed that HT had the potential to improve health, including antidepression and antioxidant effects. In this study, we proposed a strategy to explore the role and underlying mechanism of HT in improving healthspan of a Caenorhabditis elegans model. First, we found that HT significantly prolonged the lifespan without reducing fertility in worms. Second, stress resistance (oxidative stress and heat stress) was enhanced and A $\beta$ - and polyQ-induced toxicity was relieved significantly by HT treatment. Both fat deposition and age pigment accumulation were found to be significantly reduced in HT-treated worms. The locomotion in mid-late stages was improved, indicating that behavioral mobility was also significantly enhanced. Furthermore, the main components of HT were eighteen polyphenols and two terpenoids. Finally, it was found that this protective mechanism was positively correlated with the insulin/insulin-like growth factor signaling- (IIS-) dependent manner, which went through promoting the nuclear localization of DAF-16 and its downstream SOD-3 expression. These results suggested that HT had an important role in improving health, which might serve as a promising healthy tea.

\section{Introduction}

Increased age is a critical risk factor for healthspan, such as progressive decline in tissue and organ function, neurodegeneration, and metabolic disorders. Antioxidants are important strategies for delaying aging and aging-related degenerative diseases [1]. Natural active substances, in particular, are found in herbs, conferring antioxidant activities, thereby providing beneficial effects on health. According to a report by the World Health Organization, $80 \%$ of the population in developing countries rely on traditional medicine for primary health care, and $85 \%$ of traditional medicines are derived from herb extracts [2]. Recently, medicinal herbs are increasingly explored by the food industry for their health-promoting benefits either as readily available for herbal teas or as sources of additives for functional foods and drinks [3]. Thus, herbal extract-based compositions have enormous potential to be developed as a reservoir of natural antioxidant additives and innovative healthpromoting products.

Here, we explored the health effects of a Chinese traditional herbal tea (HT) made of natural herbs combined with modern technology. HT, founded in the Qing Dynasty (1828), is widely recognized as the first ancestor of herbal tea in China. HT has been widely used for a long history to treat "Shanghuo" (an unbalanced response of the body to cellular stress resulting in a series of disruptions of homeostasis) in traditional Chinese medicine [4]. In recent years, HT has been found to have various health benefits, including antistress, anti-inflammatory, and antioxidant effects [4-6]. Its main raw material is "three flowers, three grasses, and one leaf", namely, Dendranthema morifolium (Ramat.) Tzvel., 
Lonicera japonica Thunb., Plumeria rubra L. cv. Acutifolia, Glycyrrhiza uralensis Fisch, Prunella vulgaris L, Mesona chinensis Benth., and Microctis folium (the ratio of each plant species in the formula is $3.5: 3: 5: 1: 3: 5: 5)$. These seven extracts were found to be of the most promising natural antioxidants, which could improve health [7-13]. There is convincing evidence that plant-derived natural antioxidants have important potential to relieve cellular stress and maintain homeostasis [14]. Therefore, it is an attractive question that HT with antioxidant activity could improve the healthspan or not.

To investigate the bioactive substances and pharmacological studies of HT, the efficient biological model Caenorhabditis elegans was applied. C. elegans are considered to be a good model for studying aging and screening for drugs with health-promoting effects [15]. It has a number of advantages, such as a simple structure, ease of feeding, a short life cycle, rapid reproduction, a large number of offspring, and distinct molecular signaling pathways [15]. Particularly, the aging process of $C$. elegans is quite conservative in evolution. For example, C. elegans exhibits similar physiological functions to humans, such as locomotion decreasing and harmful metabolites increasing with age. In terms of the mechanism of action of aging, multiple pathways which control longevity and stress resistance are conservative from C. elegans to humans, so the knowledge obtained in C. elegans is generally applicable to mammals including mankind $[16,17]$.

Therefore, the purpose of this study was to use C. elegans, one of the most valuable and attractive model organisms, to evaluate the potential improvement of HT for lifespan and healthspan and explore the underlying mechanism of action. This result will provide a scientific basis for the benefit of HT in improving health and delaying disease onset, valuable for studies of other plant-based products.

\section{Materials and Methods}

2.1. Culture of C. elegans. C. elegans strain N2-Bristol (wildtype); strains CL4176 (dvIs27 [myo-3p::A $\beta$ (1-42)::let-851 $3^{\prime} \mathrm{UTR}+\operatorname{rol}-6($ su1006) $\left.] \mathrm{X}\right)$, CL2355 ([smg-1 ts (snb-1/A $\beta$ (1-42)/long $3^{\prime}$-UTR)]), CL2122 (unc-54 vector + mtl2::GFP), and AM140 (rmIs132 [unc-54p::Q35::YFP]); the transgenic worms CF1553 (muIs84(pAD76(sod-3::gfp))); and Escherichia coli OP50 (E. coli OP50) were obtained from the Caenorhabditis Genetics Center (CGC, University of Minnesota). TJ356 (zIs356 IV (pdaf-16-daf-16::gfp; rol-6)), VC433 (sod-3(gk235)X), and CF1038 (daf-16 (mu86)I) were presented by Prof. Qinghua Zhou (Biomedical Translational Research Institute, Jinan University, Guangdong Province, China). All strains were cultivated on nematode growth medium (NGM) agar plates seeded with E. coli OP50 at $20^{\circ} \mathrm{C}$ unless otherwise stated. Worm synchronization was described in the previously established protocol [18]. During the worm breeding period, the worms were transferred to a new plate every day, and the rest of the time, the worms were transferred to a new plate every other day.

2.2. Sample Preparation. HT stock solution was provided by Guangzhou Wanglaoji Pharmaceutical Company Limited
(Guangdong, China) and stored at $4^{\circ} \mathrm{C}$ (the analysis of various chemical constituents in HT was summarized in supplementary Table S1). The HT stock solution was mixed with E. coli OP50, the worm's food source, and dispersed on the NGM plates (the test concentrations were $10 \mathrm{mg} / \mathrm{ml}, 30 \mathrm{mg} / \mathrm{ml}$, and $50 \mathrm{mg} / \mathrm{ml}$ ). HT treatment was started from the egg stage, and the samples were harvested after 7-, 12-, and 17-day treatment unless otherwise stated.

2.3. Lifespan Assay. Lifespan assay was conducted as described before [18]. Approximately 60 individuals (strains N2, VC433, and CF1038) were treated with or without HT from the egg stage at $20^{\circ} \mathrm{C}$. The dead worms were calculated and removed daily (from the first day of hatching). Nematodes that hatch embryos in the body (so-called "internal hatching") or escape from the plates were not counted in the statistics [18]. Three replications were performed.

2.4. Fertility Assay. Fertility was measured as in the previous protocol [18]. After 48 hours with or without HT treatment, wild-type N2 worms were transferred to a new control or HT plate every 24 hours (one plate per two worms). The number of eggs laid per day was counted until no more eggs were laid. The sum of eggs per day was taken as the total number of nematode subalgebras. Three independent trials were conducted in 10 individuals per trial.

\subsection{Stress Resistance Assay}

2.5.1. Oxidative Stress Resistance Assay. As previously described [19], treated on a medium with or without HT for ten days, the worms were transferred to oxidative stress plates with additional $10 \mathrm{mM}$ paraquat. The survival was monitored every 12 hours. Three independent biological replicates were performed with a total of 180 worms.

2.5.2. Heat Stress Resistance Assay. As previously described [19], wild-type N2 worms were treated with or without HT from the egg stage at $20^{\circ} \mathrm{C}$ for 4 days and then were transferred to a $35^{\circ} \mathrm{C}$ culture environment. The number of dead worms was recorded every hour. Sixty worms were used in each experiment for three repetitive tests.

\subsection{Neuroprotective Assay against A $\beta$-Induced Toxicity}

2.6.1. A $\beta$-Induced Paralysis Assay. CL4176 strains were cultivated in NGM plates containing HT or vehicle (control) for 36 hours at $16^{\circ} \mathrm{C}$ and then transferred to $25^{\circ} \mathrm{C}$ to induce $\mathrm{A} \beta$ expression as described previously with minor modifications [20]. The worms were categorized as paralyzed, which did not move or just moved the head when gently touched with a platinum loop. Paralysis was scored at 2-hour intervals until all worms were paralyzed. The assay was performed for three biological replicates with 60 worms per trial.

2.6.2. A $\beta$-Induced Chemotactic Dysfunction Assay. A $\beta$ induced chemotaxis assay was performed as previously described [21]. The eggs of CL2355 or CL2122 (a control strain) transgenic strains were treated with or without HT 
at $16^{\circ} \mathrm{C}$ for $36 \mathrm{~h}$ and then at $25^{\circ} \mathrm{C}$ for $36 \mathrm{~h}$.Approximately 60 worms were placed in the center of a chemotaxis agar plate $(9 \mathrm{~cm})$. Before the placement, $1 \mu \mathrm{l}$ of $0.25 \mathrm{M}$ sodium azide along with $1 \mu \mathrm{l}$ of odorant $(0.1 \%$ benzaldehyde in $100 \%$ ethanol) was added to one side of the plate ("attractant" spot). On the opposite spot, $1 \mu \mathrm{l}$ of control odorant (100\% ethanol) and $1 \mu \mathrm{l}$ of $0.25 \mathrm{M}$ sodium azide were added. The plates were incubated for $1 \mathrm{~h}$ at $25^{\circ} \mathrm{C}$, and then, the number of worms in each quadrant (up to $2 \mathrm{~cm}$ radius from the spots) was scored. The chemotaxis index (CI) was calculated $(\mathrm{CI}=$ (number of worms in attractant quadrants - number of worms in control quadrants) / total number of scored worms).

2.7. polyQ-Dependent Paralysis Assay. Cultured as described in the lifespan assay, AM140 transgenic worms cultured with or without HT were defined as paralyzed if they failed to move forward upon the tail prodding as previously described [22]. The assay was performed for three independent trials with 60 worms per trial.

2.8. Age Pigment Fluorescence Assay. To assess the accumulation of age pigment, age-synchronized worms (N2 strain) were treated with or without HT for 7, 12, and 17 days. Subsequently, the worms were anesthetized and mounted on a glass slide. Animals were selected randomly to submit to fluorescence microscopy with a GFP filter (Axio Imager Z2, Carl Zeiss AG, Jena, Germany). Considering that it is easy to confuse the strong near-death green autofluorescence with the increases in GFP in worms by green autofluorescence (through the GFP filter set) [23], the nematode individuals selected had strong vitality and were able to respond quickly to stimulation. Images were taken from at least 15 worms per group with a $10 \mathrm{x}$ objective. ImageJ software (NIH Image) was used to analyze the average pixel intensity of each worm in four independent trials.

2.9. Intestinal Fat Deposition Assay. As previously established, Oil Red $\mathrm{O}$ staining was used to determine fat content, but the freeze-thaw steps were omitted [24]. Briefly, worms were fixed in $4 \%$ paraformaldehyde, dehydrated in $60 \%$ isopropanol, and then stained with Oil Red O staining solution for 12 hours in the black with gentle rocking. Deposits of intestinal fat were imaged 40 times with an SLR camera (Canon Eos 5D Mark II) and quantified using ImageJ software (NIH Image). The assay was performed in three independent trials with at least 45 wild-type N2 worms.

2.10. Locomotion Assay. Locomotion assay was performed in three different life stages (the early, middle, and mid-late) as described [25]. Worms were observed and classified (classes $\mathrm{A}, \mathrm{B}$, and C) by a stereomicroscope according to the response of worms to stimuli. The movement behavior of 60 worms was detected after a one-minute recovery period. Three independent experiments were performed.

2.11. Pharyngeal Pumping Assay. The pharyngeal pumping frequency was measured by monitoring the movement of the pharynx terminal bulb under a stereomicroscope for
30 s (15 worms). Three independent experiments were performed in duplicate.

2.12. E. coli OP50 Bacterial Growth Assay. The bacterial growth assay of $E$. coli OP50 was performed as described previously [24]. The determination was set at OD600 for $12 \mathrm{~h}$ $(n=3)$.

2.13. Sample Characterization by Liquid Chromatography Coupled with Quadrupole Time of Flight Mass Spectrometry (LC-Q-TOF-MS/MS) Analysis. LC-Q-TOF-MS/MS analysis was used to investigate the sample components. The chromatographic system consisted of an Eclipse Plus C18 $(2.0 \times 100 \mathrm{~mm}, 1.8 \mu \mathrm{m}$, Agilent $)$ with a binary pump, degasser, column oven, and autosampler performed on a Uplc12906540B Q-TOF (Agilent Technologies, Palo Alto, CA, USA). Separation of HT was achieved on a 30 min linear gradient of acetonitrile (A) and $0.2 \%$ formic acid in ultrapure water (B), increasing from 5 to $95 \%$ B, with a 2 min hold at $95 \%$ B and a 4 min postrun at $10 \% \mathrm{~B}$. The injection volume was $1 \mu \mathrm{l}$, and the flow rate was $0.4 \mathrm{ml} / \mathrm{min}$ at $40^{\circ} \mathrm{C}$. For the online TOF-MS and TOF-MS/MS analysis, experimental operation parameters were set as follows: nozzle voltage, $500 \mathrm{~V}$; skimmer, $65 \mathrm{~V}$; gas flow, $8 \mathrm{l} / \mathrm{min}$; gas temp, $300^{\circ} \mathrm{C}$; and nebulizer, 45 psi. The collision energy was set at $0 \mathrm{eV}$ for negative and positive ion mode. The mass scan was over the range of $\mathrm{m} / z$ 105-1700 for both modes. Data analysis was carried out using the Agilent Mass Hunter Qualitative software (version B.07.00). Accurate mass scan data were mined using the find by molecular feature, find by formula, and find by targeted MS/MS and molecular formula generator algorithms.

2.14. SOD-3::GFP Visualization and Quantification Assay. To assess SOD-3::GFP expression, CF1553 worms were treated with or without HT for 96 hours as previously described [25]. The fluorescence of SOD-3::GFP was imaged using a fluorescence microscope with suitable filter and a 10x objective (Axio Imager Z2, Carl Zeiss AG, Jena, Germany) and analyzed using ImageJ software (NIH Image) in the whole body region ( $>15$ worms/trial, with a total of 3 trials).

2.15. DAF-16::GFP Visualization and Delocalization Assay. To determine the subcellular localization of DAF-16::GFP, TJ356 worms were treated with or without HT for 96 hours as previously described [26]. The fluorescence of DAF16::GFP was imaged using a Leica TCS SP8 (Buffalo Grove, IL) laser scanning confocal microscope with 100-fold magnification (excitation wavelength: $488 \mathrm{~nm}$; emission wavelength: $510-550 \mathrm{~nm})$. At 30 worms per treatment were scored as cytosolic, intermediate, and nuclear. The number of worms in each class was calculated from at least three biological replicates.

2.16. Statistical Analysis. It was considered to be a statistically significant difference if $p<0.05$ applying one-way ANOVA followed by post hoc comparison (Tukey-Kramer test) for multiple comparison and independent samples $t$-test for two group comparisons (SPSS software, version 16). Survival curves were analyzed using Mantel-Cox log-rank test by using GraphPad Prism (GraphPad Prism, version 5.00 for 


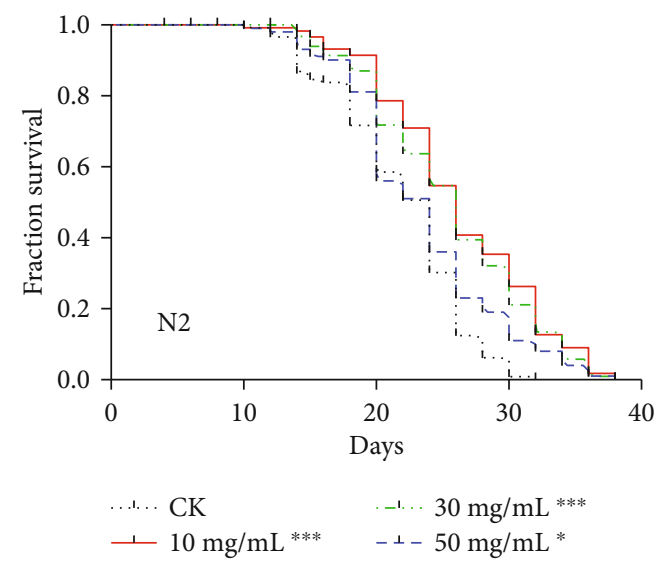

(a)

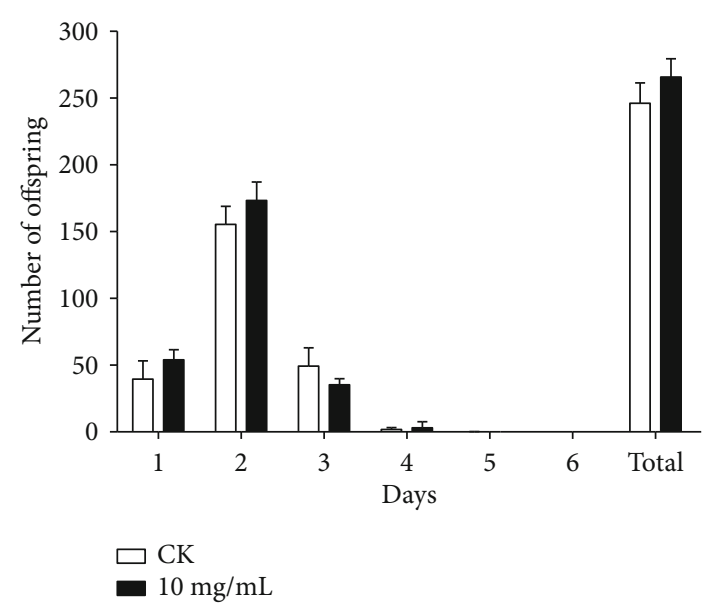

(b)

FIGURE 1: Effects of HT on lifespan and fertility of C. elegans: (a) lifespan curves of HT on N2 worms $\left({ }^{*} p<0.05,{ }^{* *} p<0.01\right.$, and ${ }^{* * *} p<0.001$ compared with the CK group, the log-rank test); (b) the effect of HT on worm fecundity at the optimum longevity dose. The results are presented as the mean $\pm \mathrm{SD}$.

TABLe 1: Statistical analysis of survival time of C. elegans.

\begin{tabular}{|c|c|c|c|c|c|}
\hline Genotype & Treatment $(\mathrm{mg} / \mathrm{ml})$ & Mean time $e^{(1)}$ & Maximum time ${ }^{(2)}$ & $\%$ effect $^{(3)}$ & $p$ value $^{(4)}$ \\
\hline \multirow{8}{*}{ N2 } & 0 & $21.70 \pm 1.86^{\mathrm{a}}$ & $29.50 \pm 1.91^{\mathrm{a}}$ & 1 & 1 \\
\hline & 10 & $26.09 \pm 0.53^{\mathrm{b}}$ & $37.00 \pm 1.15^{\mathrm{b}}$ & 20 & $<0.0001$ \\
\hline & 30 & $25.28 \pm 2.41^{\mathrm{ab}}$ & $35.50 \pm 2.52^{\mathrm{b}}$ & 16 & $<0.0001$ \\
\hline & 50 & $23.39 \pm 1.43^{\mathrm{ab}}$ & $34.50 \pm 3.42^{\mathrm{ab}}$ & 8 & 0.0260 \\
\hline & 0 (paraquat) & $4.43 \pm 0.26^{\mathrm{a}}$ & $7.50 \pm 0.50^{\mathrm{a}}$ & \multirow{2}{*}{12} & \multirow{2}{*}{0.0034} \\
\hline & 10 (paraquat) & $4.95 \pm 0.09^{\mathrm{b}}$ & $9.00 \pm 0.50^{\mathrm{b}}$ & & \\
\hline & 0 (heat) & $15.36 \pm 0.28^{\mathrm{a}}$ & $21.67 \pm 0.58^{\mathrm{a}}$ & \multirow{2}{*}{15} & \multirow{2}{*}{$<0.0001$} \\
\hline & 10 (heat) & $17.28 \pm 0.84^{\mathrm{b}}$ & $23.33 \pm 0.58^{\mathrm{b}}$ & & \\
\hline \multirow{2}{*}{ CL4176 } & 0 & $10.02 \pm 0.69^{\mathrm{a}}$ & $14.33 \pm 0.58^{\mathrm{a}}$ & \multirow{2}{*}{16} & \multirow{2}{*}{$<0.0001$} \\
\hline & 10 & $11.67 \pm 0.34^{\mathrm{b}}$ & $15.33 \pm 0.58^{\mathrm{b}}$ & & \\
\hline \multirow{2}{*}{ AM140 } & 0 & $11.95 \pm 0.22^{\mathrm{a}}$ & $15.67 \pm 1.53^{\mathrm{a}}$ & \multirow{2}{*}{9} & \multirow{2}{*}{$<0.0001$} \\
\hline & 10 & $13.08 \pm 0.29^{\mathrm{b}}$ & $17.67 \pm 0.58^{\mathrm{b}}$ & & \\
\hline \multirow{2}{*}{ VC433 } & 0 & $17.57 \pm 0.22$ & $28.33 \pm 0.58$ & \multirow[b]{2}{*}{ l } & \multirow{2}{*}{ l } \\
\hline & 10 & $17.08 \pm 0.25$ & $28.00 \pm 1.00$ & & \\
\hline \multirow{2}{*}{ CF1038 } & 0 & $16.65 \pm 0.12$ & $26.33 \pm 1.15$ & \multirow{2}{*}{ I } & \multirow{2}{*}{ l } \\
\hline & 10 & $16.34 \pm 0.32$ & $26.33 \pm 0.58$ & & \\
\hline
\end{tabular}

(1) Mean time $=(1 / n) \sum_{j}\left(\left(x_{j}+x_{j+1}\right) x_{j}+x_{j+1} / 2\right) d j$, where $j$ is the age category, $d_{j}$ is the number of worms that died in the age interval $\left(x_{j}, x_{j+1}\right)$, and $n$ is the total number of worms. ${ }^{(2)}$ The maximum time is the time at which fraction survival equals $0 \%{ }^{(3)} \%$ effect was calculated by $(T-C) / C * 100$, where $T$ is the mean time of worms treated with $\mathrm{HT}$ and $C$ is the mean time of control. ${ }^{(4)} p$ value was calculated using the log-rank test by comparing the treated group with control.

Windows). The two-stage procedure was used when there was an intersection in the survival curve [27]. All data are expressed as mean $\pm S D$, calculated in three independent experiments. Different letters in columns indicate that the values are significantly different $(p<0.05)$. In the survival curve, ${ }^{*} p<0.05,{ }^{* *} p<0.01$, and ${ }^{* * *} p<0.001$ compared with the control check $(\mathrm{CK})$ group.

\section{Results and Discussion}

3.1. Effects of HT on Lifespan of C. elegans. First, we studied the effect of HT on the lifespan of C. elegans. Interestingly, the lifespan curves of the three test concentrations $(10 \mathrm{mg} / \mathrm{ml}, 30 \mathrm{mg} / \mathrm{ml}$, and $50 \mathrm{mg} / \mathrm{ml})$ all showed a significant right shift compared to those of the control group $(p<0.0001$ by the log-rank test for $10 \mathrm{mg} / \mathrm{ml}$ and $30 \mathrm{mg} / \mathrm{ml}, p=0.0260$ by the log-rank test for $50 \mathrm{mg} / \mathrm{ml}$ ), suggesting that the HT sample had the benefit of extending lifespan in C. elegans (Figure 1(a)). Moreover, the most significant longevity effect of HT was found at the concentration of $10 \mathrm{mg} / \mathrm{ml}$, which increased the mean lifespan by $20 \%$ (26.09 days vs. 21.70 days) and extended the maximum lifespan by 7.50 days (37.00 days vs. 29.50 days) (Table 1). It was evident that HT had the potential to prolong the lifespan of C. elegans. 

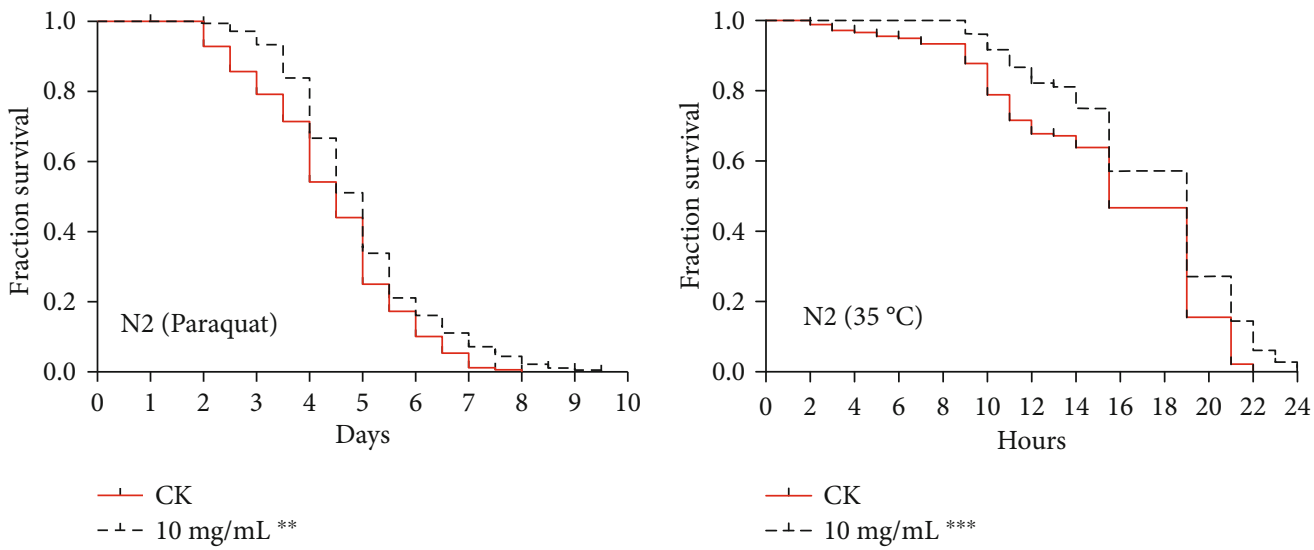

(a)

(b)
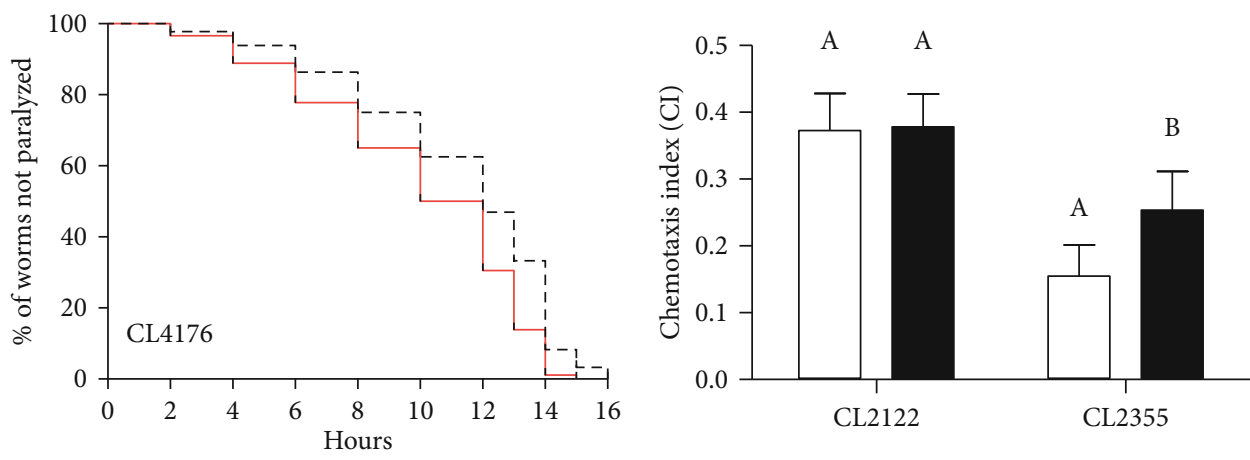

$\rightarrow \mathrm{CK}$

$-\perp-10 \mathrm{mg} / \mathrm{mL}^{* * *}$

$$
\begin{aligned}
& \square \mathrm{CK} \\
& 10 \mathrm{mg} / \mathrm{mL}
\end{aligned}
$$

(c)

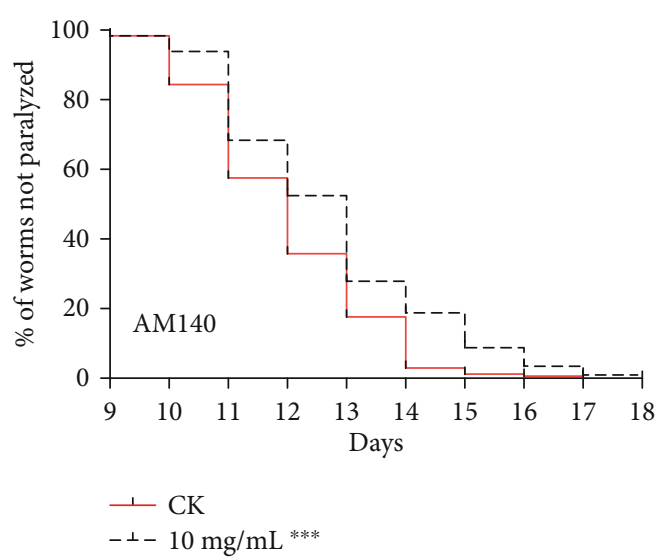

(e)

FIgURE 2: Effects of HT on oxidative, heat, and proteotoxic stresses in C. elegans: (a) survival curve of worms exposed to paraquat-induced oxidative stress after incubation with HT; (b) survival curve of worms exposed to $35^{\circ} \mathrm{C}$ for heat stress testing after the nematodes were pretreated with $\mathrm{HT}$ at $20^{\circ} \mathrm{C}$ for 96 hours; (c) paralysis curve of CL4176 worms; (d) the A $\beta$-induced chemotactic dysfunction index after treatment of HT; (e) paralysis curve of AM140 worms. All data are presented as the mean \pm SD of at least three independent experiments. Different letters above bars indicate a significant difference at $p<0.05$ and ${ }^{* *} p<0.01$ and ${ }^{* * *} p<0.001$ compared with the CK group in the survival curve.

Since the optimal lifespan extension effect of HT was observed at the dose of $10 \mathrm{mg} / \mathrm{ml}$, we further used the concentration to investigate and test whether HT had an adverse effect on the number of progenies. As shown in Figure 1(b), there was no significant difference in the daily number of offspring and total number of offspring in worms treated with control or HT, indicating that HT neither delayed nor inhibited fertility. So HT did not produce obvious reprotoxicity to worms and the beneficial effects of HT on lifespan might be independent of reproductive capacity in C. elegans at the dose of $10 \mathrm{mg} / \mathrm{ml}$. 


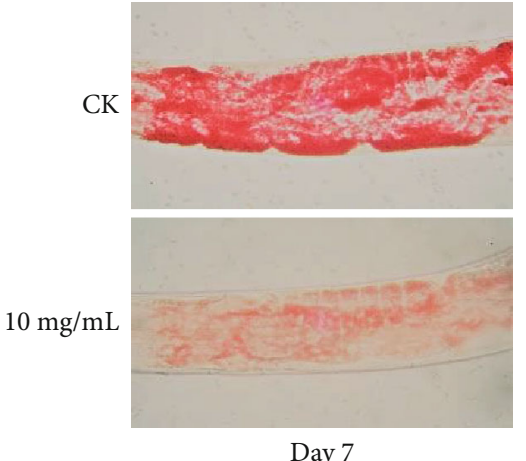

(a)

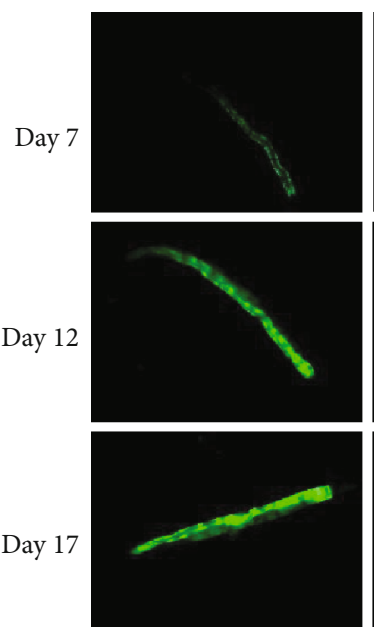

CK

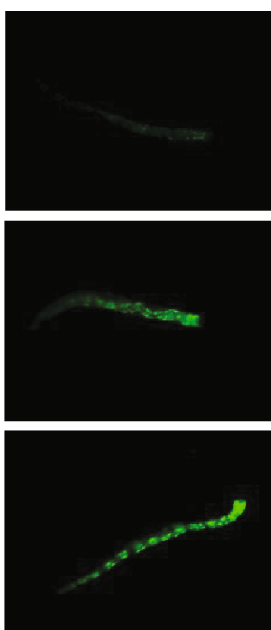

$10 \mathrm{mg} / \mathrm{mL}$

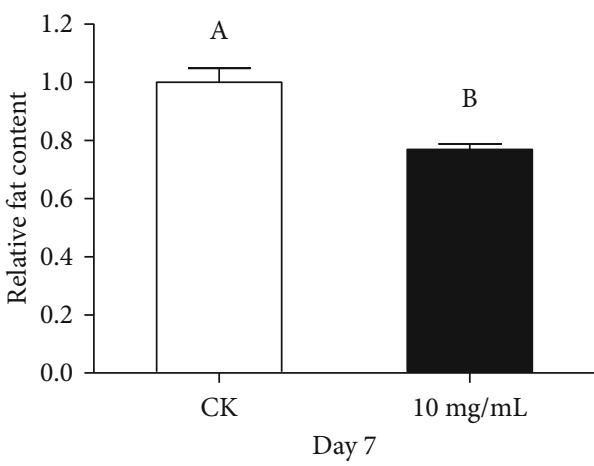

(b)

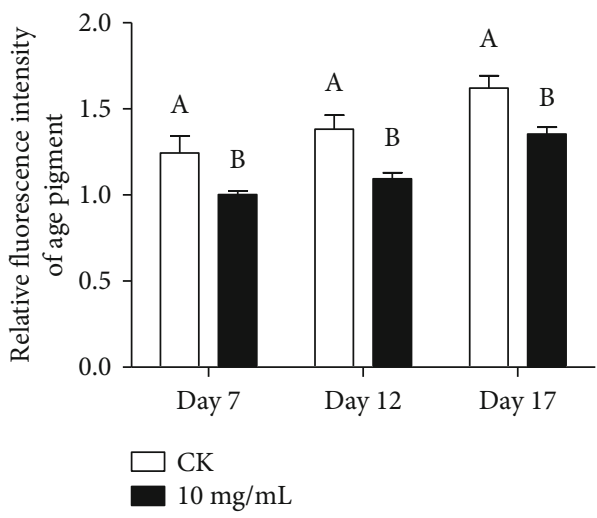

(d)

FIGURE 3: Effects of HT on the accumulation of fat and age pigments in C. elegans: (a) representative Oil Red O staining of nematode intestinal fat for control group and HT group; (b) the relative intensity of Oil Red O staining in the HT group and the control group; (c) the typical age pigment fluorescence images of HT group and control group in the early, middle, and mid-late stages (days 7, 12, and 17, respectively); (d) the effect of HT on the age pigment of N2. Bars represent mean \pm SD of at least three experiments, and different letters above bars indicate a significant difference at $p<0.05$.

\subsection{Effects of HT on Oxidative, Heat, and Proteotoxic Stresses} in C. elegans. A large body of credible evidence suggested that longevity was often accompanied by increased resistance to environmental stress, and stress resistance might be a determining factor in the lifespan of C. elegans [28]. Firstly, we investigated two stress conditions to comprehensively evaluate stress tolerance, which were paraquat-induced oxidative stress and heat stress. Considering that a large number of the matricidal death ("bagging") of nematodes will happen if the nematodes are exposed to paraquat at the reproductive period [29], oxidative stress was carried out after the reproductive period (the 10th day) to avoid the matricidal death. Heat stress was measured on the 4th day of the life cycle, which is the peak period of adult worm vitality. The survival curve of HT treatment was different from that of the control group ( $p=0.0034$ and $p<0.0001$ by the log-rank test for oxidative stress and heat stress, respectively, Figures 2(a) and 2(b)). In oxidative stress and heat stress protection assay, HT treatment significantly increased the mean lifespan (prolongation of 12 and 15\%, respectively, Table 1) and the maximum lifespan (extended from 21.67 to 23.35 days and from 7.50 days to 9.00 days, respectively, Table 1). It suggested that HT had the potential to protect against stress.

It is increasingly clear that protein misfolding, which is caused by cellular stress, leads to cell dysfunction [30]. When these events occur in neurons, they may lead to neurodegenerative diseases over time [30]. Secondly, to determine the effect of HT on proteotoxic stress, we applied transgenic $C$. elegans CL4176, which expresses an aggregating amyloid-beta (3-42) peptide $(\mathrm{A} \beta(3-42))$ in muscle tissue [31]. In humans, $A \beta(3-42)$ aggregation is considered to be a key pathological factor in Alzheimer's disease [32]. We found that the HT treatment group exhibited a significantly right-shifted paralysis curve in 


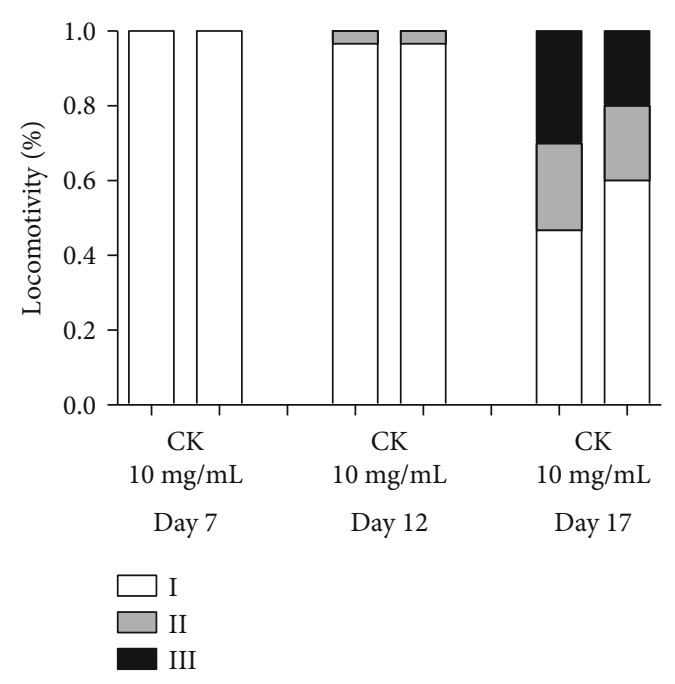

(a)

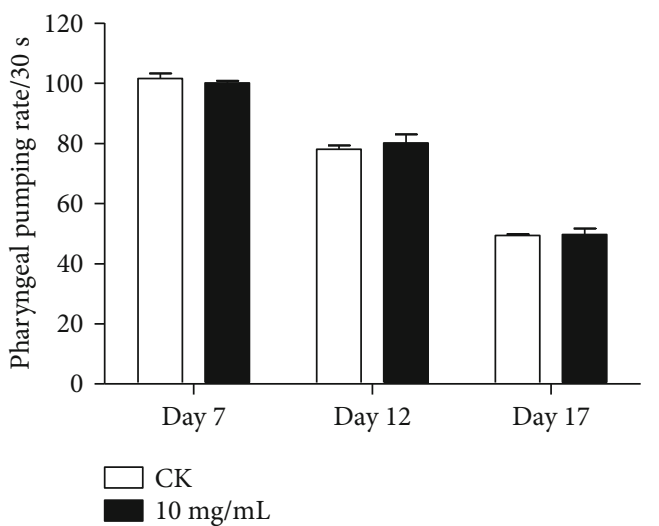

(b)

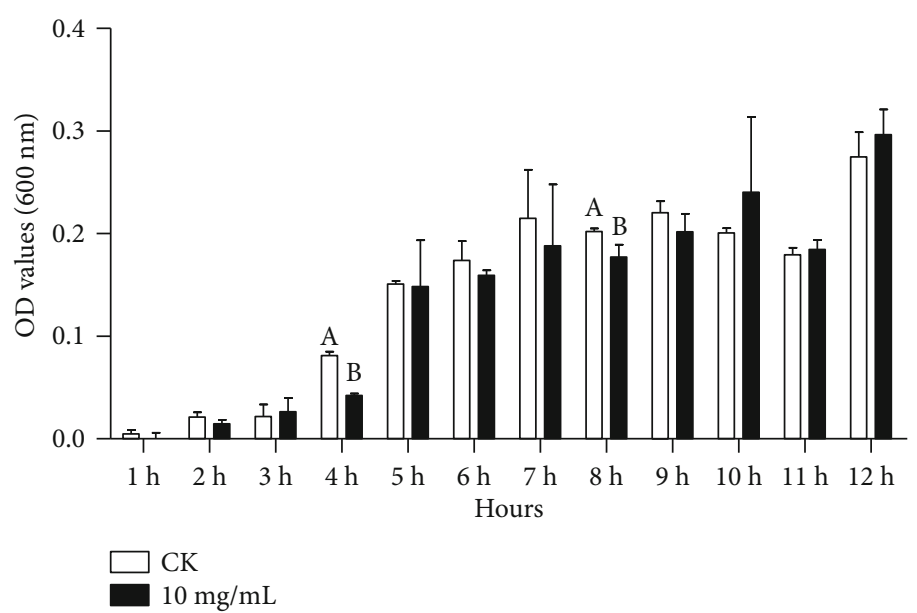

(c)

Figure 4: Effects of HT on behavioral mobility C. elegans: (a) the effect of HT on the age-related decline in exercise of N2 after 7, 12, and 17 days of treatment. Individuals were classified according to their locomotion: a free movement, B-stimulated motion, and C-stimulated weak motion; (b) the effect of HT on the frequency of pharyngeal pumping of N2; (c) the curve of E. coli OP50 bacterial growth after treatment of $\mathrm{HT}$. All data are expressed as mean $\pm \mathrm{SD}$ of at least three experiments.

CL4176 ( $p<0.0001$ by the log-rank test, Figure $2(\mathrm{c}))$, suggesting a significant delay in the paralysis time overall. HT treatment significantly delayed the mean paralysis time by $16 \%$, with the maximum paralysis time from 14.33 to 15.44 hours in CL4176 (Table 1). Subsequently, we studied the effect of HT on A $\beta$-induced neurotoxicity using CL2355 strain expressing human $\mathrm{A} \beta_{1-42}$ in neuronal cells (a phenomenon leading to chemotactic dysfunction). As shown in Figure 2(d), the CI of the blank strain CL2122 (no $\mathrm{A} \beta$ expression) was lower than that of the untreated $\mathrm{A} \beta$ strain CL2355. These data demonstrated that CL2355 nematodes suffered more damage in neurons sensitive to the attractant benzaldehyde. Strikingly, the CI of the CL2355 strain treated with HT was significantly higher than that of the untreated CL2355. These results indicated that HT had a neuroprotective effect against $\mathrm{A} \beta$-induced paralysis in $C$. elegans. Furthermore, another transgenic nematode model AM140 was applied. The AM140 worm expresses polyglutamine (polyQ) protein in body wall muscle cells, which is characteristic of several neurological diseases [32]. A similar inhibitory paralysis effect was also observed in AM140 (Figure 2(e)). In polyQ-dependent paralysis, not only a significantly different paralysis curve was exhibited in the HT-treated AM140 worms, but also the mean paralysis time and maximum paralysis time were significantly prolonged (Figure 2(e) and Table 1). Collectively, HT conferred stress resistance against different stressors, including oxidative stress, heat stress, and proteotoxic stress, which might alleviate protein misfolding and maintain protein homeostasis.

3.3. Effects of HT on the Reduction of Fat Accumulation and Age Pigment in C. elegans. The prevalence of obesity has risen sharply, adversely affecting longevity and health throughout the life cycle [33]. Since excessive fat accumulation has a serious impact on the quality of life, we investigated the effect of HT on the fat accumulation of $C$. elegans. The staining intensity of HT-treated nematodes 
TABLE 2: Tentative identification of chemical constituents of HT by UPLC/Q-TOF-MS/MS.

\begin{tabular}{|c|c|c|c|c|c|c|c|}
\hline $\mathrm{RT}(\min )$ & Mass & Abund & Percentage composition & Formula & Diff $(\mathrm{ppm})$ & Significant IonMz & Tentative identification \\
\hline 7.045 & 516.1273 & 354272 & 6.00944 & $\mathrm{C}_{25} \mathrm{H}_{24} \mathrm{O}_{12}$ & 1.11 & 515.1202 & Isomeric di-O-caffeoylquinic acid \\
\hline 7.625 & 404.1326 & 150559 & 2.5539 & $\mathrm{C}_{17} \mathrm{H}_{24} \mathrm{O}_{11}$ & 1.72 & 403.1253 & Secoxyloganin \\
\hline 8.569 & 610.1543 & 160513 & 2.722748 & $\mathrm{C}_{27} \mathrm{H}_{30} \mathrm{O}_{16}$ & 1.56 & 609.1472 & Rutin \\
\hline 8.793 & 464.0954 & 150392 & 2.551067 & $\mathrm{C}_{21} \mathrm{H}_{20} \mathrm{O}_{12}$ & -0.14 & 465.1029 & Isoquercitrin \\
\hline 8.901 & 448.1006 & 186538 & 3.164204 & $\mathrm{C}_{21} \mathrm{H}_{20} \mathrm{O}_{11}$ & 0.01 & 449.1079 & Trifolin \\
\hline 8.909 & 462.0797 & 102139 & 1.732562 & $\mathrm{C}_{21} \mathrm{H}_{18} \mathrm{O}_{12}$ & -0.21 & 463.0872 & Scutellarin \\
\hline 8.909 & 154.0266 & 102139 & 1.732562 & $\mathrm{C}_{7} \mathrm{H}_{6} \mathrm{O}_{4}$ & -0.1 & 463.0872 & Protocatechuic acid \\
\hline 9.273 & 594.1594 & 247027 & 4.190266 & $\mathrm{C}_{27} \mathrm{H}_{30} \mathrm{O}_{15}$ & 1.53 & 593.1523 & 1-Hydroxybenzotriazole \\
\hline 9.274 & 198.0528 & 159629 & 2.707753 & $\mathrm{C}_{9} \mathrm{H}_{10} \mathrm{O}_{5}$ & -0.02 & 595.1658 & Syringic acid \\
\hline 9.298 & 516.1279 & 602707 & 10.22359 & $\mathrm{C}_{25} \mathrm{H}_{24} \mathrm{O}_{12}$ & 2.13 & 515.1208 & Isochlorogenic acid C \\
\hline 9.431 & 138.0317 & 446001 & 7.56542 & $\mathrm{C}_{7} \mathrm{H}_{6} \mathrm{O}_{3}$ & 0.17 & 137.0244 & Protocatechualdehyde \\
\hline 9.513 & 516.1276 & 243848 & 4.136341 & $\mathrm{C}_{25} \mathrm{H}_{24} \mathrm{O}_{12}$ & 1.6 & 515.1204 & Isochlorogenic acid A \\
\hline 9.803 & 718.1547 & 173276 & 2.939244 & $\mathrm{C}_{36} \mathrm{H}_{30} \mathrm{O}_{16}$ & 1.81 & 717.1476 & Salvianolic acid B \\
\hline 9.944 & 516.1277 & 666790 & 11.31062 & $\mathrm{C}_{25} \mathrm{H}_{24} \mathrm{O}_{12}$ & 1.7 & 515.1206 & Isochlorogenic acid B \\
\hline 10.11 & 360.0859 & 755269 & 12.81147 & $\mathrm{C}_{18} \mathrm{H}_{16} \mathrm{O}_{8}$ & 3.75 & 719.1636 & Rosmarinic acid \\
\hline 10.59 & 418.127 & 192063 & 3.257924 & $\mathrm{C}_{21} \mathrm{H}_{22} \mathrm{O}_{9}$ & 1.38 & 417.1198 & Liquiritin \\
\hline 10.773 & 446.0847 & 275568 & 4.674401 & $\mathrm{C}_{21} \mathrm{H}_{18} \mathrm{O}_{11}$ & -0.4 & 447.0921 & Baicalin \\
\hline 10.797 & 718.1545 & 229366 & 3.890686 & $\mathrm{C}_{36} \mathrm{H}_{30} \mathrm{O}_{16}$ & 1.56 & 717.1473 & Salvianolic acid E \\
\hline 11.477 & 592.1801 & 148132 & 2.512731 & $\mathrm{C}_{28} \mathrm{H}_{32} \mathrm{O}_{14}$ & 1.58 & 637.1786 & Linarin \\
\hline 14.94 & 822.4041 & 549030 & 9.313078 & $\mathrm{C}_{42} \mathrm{H}_{62} \mathrm{O}_{16}$ & 0.32 & 823.4114 & Glycyrrhizic acid \\
\hline
\end{tabular}

was significantly weaker than that of the control group, as monitored by Oil Red $\mathrm{O}$ staining (Figure 3(a)). Using ImageJ quantitative analysis of the staining intensity, the result showed a $23 \%$ reduction in the HT treatment group (Figure 3(b)). It was suggested that HT had the potential to reduce fat accumulation in C. elegans.

A conservative feature of aging is the accumulation of age pigments with age [34]. These fluorescent compounds include autofluorescence lipofuscin and advanced glycation end products [34]. The accumulation of age pigments, the biomarkers of aging, and the healthspan of $C$. elegans were comprehensively evaluated in the different stages (days 7 , 12 , and 17 represented the early, middle, and mid-late stages, respectively). In wild-type animals, age pigments accumulated with age (Figures 3(c) and 3(d)). However, HT treatment significantly alleviated the deposition of age pigments by $19 \%, 21 \%$, and $16 \%$ at different stages, respectively (Figures 3(c) and 3(d)).

3.4. Effects of HT on Behavioral Mobility in C. elegans. We further investigated the effects on behavioral mobility. First, the locomotion was investigated according to three levels. And we focused on the locomotion in the early, middle, and mid-late stages. On day 17, compared to the control group, HT treatment group increased significantly the number of free-moving worms but decreased the number of individuals which could not complete the systemic movement after stimulation (Figure 4(a)). Second, the pharynx is the main organ of worm feeding, so its muscle contractility affects the absorption and metabolism of bacteria and HT [35]. We further investigated the frequency of pharyngeal pumping, a marker for food intake, in three different periods.
Strikingly, no significant difference was observed between the treatment and the control groups (Figure 4(b)). Considering the antibacterial potential of $\mathrm{HT}$, we speculated that the absence of improvement in the pharyngeal pumping frequency of worms was due to the growth inhibition of E. coli OP50, as a marker for food availability in C. elegans. However, this speculation was rejected since the result showed that growth of $E$. coli OP50 had not been affected after 9 hours (Figure 4(c)). Obviously, HT treatment could significantly improve the locomotion of nematodes but had no effect on feeding behavior as previously described by Hibiscus sabdariffa L. extract [36]. It also showed that the calorie restriction effect might not be the key reason for antiaging of HT.

3.5. Effect of HT on Delaying Aging Might Be Attributed to Its Components. To further analyze the composition of HT, LCQ-TOF-MS/MS was performed. Representative total ion chromatograms (TIC) of HT are shown in Figure S1. Twenty compounds were tentatively identified in HT according to the previous reports (eighteen polyphenols and two terpenoids, Table 2) [5]. The main components were polyphenols, such as rosmarinic acid, isochlorogenic acid $\mathrm{B}$, isochlorogenic acid $\mathrm{C}$, glycyrrhizic acid, and protocatechualdehyde. In previous investigations [37], we found that rosmarinic acid improved antioxidant activity and healthspan in C. elegans. Isochlorogenic acids, as bioactive components of Lonicera japonica Thunb., has a wide range of biological activities, including antioxidant, antibacterial, and antiviral [38]. Strikingly, studies had shown that isochlorogenic acid B had a protective effect on liver fibrosis in mice with nonalcoholic steatohepatitis. And 


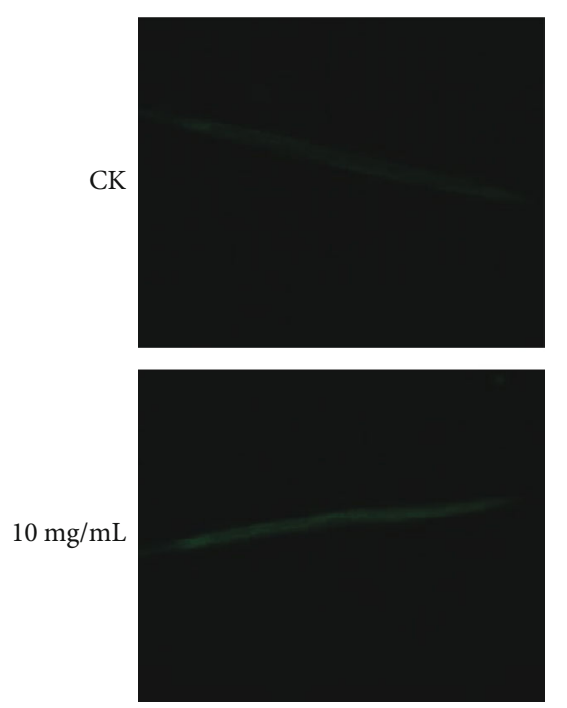

96 hours

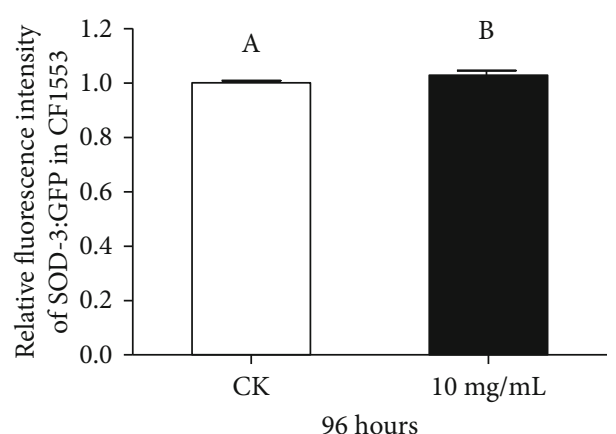

(b)

(a)

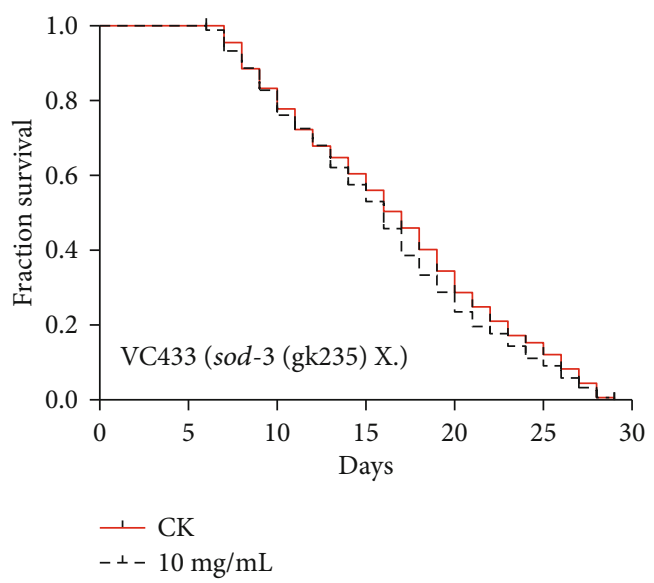

(c)

FIGURE 5: Effects of HT on the SOD-3 expression in C. elegans: (a) representative fluorescence images of SOD-3::GFP in CF1553 worms with or without HT treatment; (b) the effect of HT treatment on the expression of SOD-3::GFP in CF1553. Bars represent mean \pm SD, and the different letters above bars indicate a significant difference at $p<0.05$; (c) lifespan curves of HT on sod-3 mutant worms.

isochlorogenic acid $\mathrm{C}$ was found to prevent enterovirus 71 infection by regulating the redox homeostasis of glutathione [39]. Glycyrrhizic acid and protocatechualdehyde had been proven to have powerful antioxidant effects, which are considered to be promising potential human antiaging compounds [40, 41]. In general, natural antioxidants are considered an effective strategy for antiaging. Several studies demonstrated that supplementing with antioxidants from natural sources, such as plant extracts, could prolong the lifespan of many model organisms [42]; however, whether the effectiveness of antioxidants could be used as an indicator of longevity stills remain unclear. For example, Pun et al. pointed out that there was no intrinsic relationship between antioxidant activity and longevity of nematodes exposed to six plant extracts [43]. In this study, we also wondered whether the HT-mediated longevity was associated with increased antioxidant activity in nematodes.
We found that HT enhanced the nematode resistance to paraquat-induced oxidative stress. So the effect of HT on delaying aging might be attributed to the excellent oxidative stress tolerance ability and health improvement potential of its components. But the topic of whether HT prolonged lifespan by improving antioxidant defense mechanisms deserved further discussion.

3.6. Effects of HT on Protein Expression of SOD-3::GFP in C. elegans. SOD-3, a mitochondrial Fe/Mn superoxide dismutase, uses ROS as a substrate to convert substances that produce cell damage into safer compounds [44]. To explore the contribution of SOD-3 to the health benefits mediated by HT, we applied a SOD-3::GFP reporter strain CF1553 for visualization of SOD-3 expression. The HT-treated group showed a higher intensity of SOD-3::GFP expression than the control group (Figures 5(a) and 5(b)). To further verify whether gene sod-3 


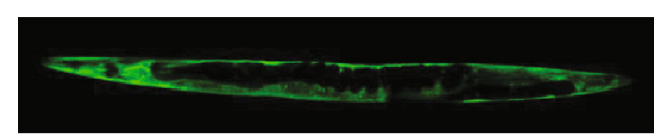

Cytosolic

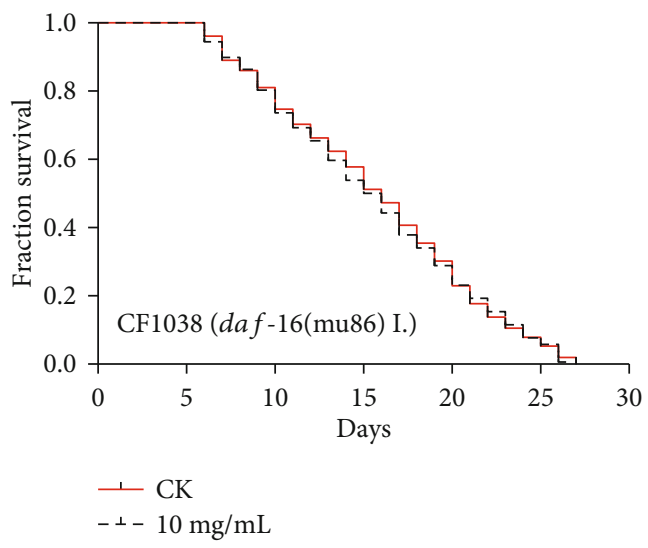

(a)

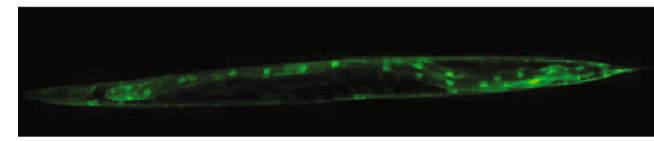

Intermediate

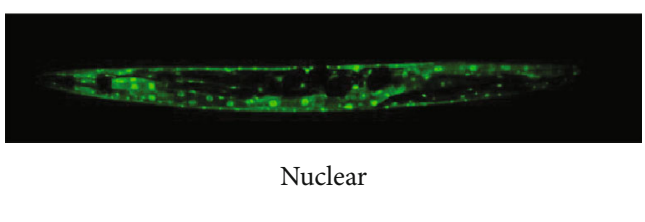

(b)

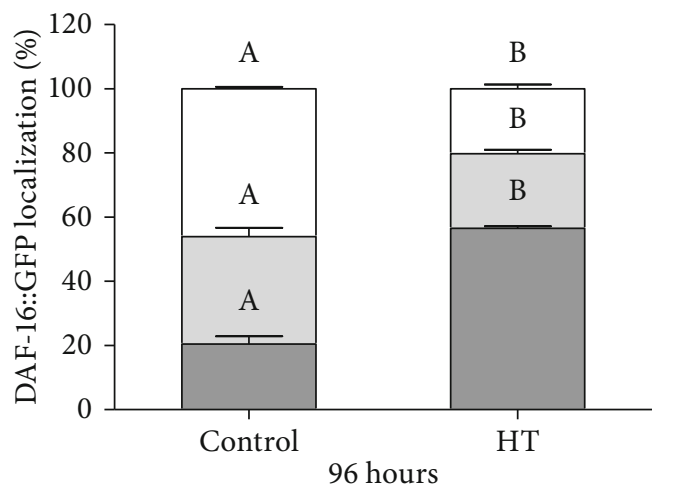

Cytosolic

Intermediate

Nuclear

(c)

FIgure 6: Effects of HT on the DAF-16 expression in C. elegans: (a) lifespan curves of HT on daf-16 mutant worms; (b) three typical distributional fluorescence images of DAF-16 in the transgenic C. elegans strain TJ356, including cytosol localization, intermediate localization, and nuclear localization; (c) the effect of HT treatment on the subcellular distribution of DAF-16 in TJ356. Bars represent mean $\pm \mathrm{SD}$, and the different letters above bars indicate a significant difference at $p<0.05$.

was involved with HT-mediated longevity, we measured the lifespan of sod-3 null mutant VC433. Consistent with this result, our results showed that the lifespan extension was completely abolished in the sod-3 mutant strain, revealing a SOD-3-dependent mechanism in HT-mediated longevity (Figure 5(c)). Therefore, we speculated that the intracellular stress alleviation of HT was positively correlated with increased SOD-3 expression, which lowered the intrinsic pressure of the cells to maintain homeostasis [45].

3.7. Effects of HT on the Subcellular Localization of DAF-16 in C. elegans. In C. elegans, the transcription factor DAF-16, the ortholog of mammalian FoxO3a (also known as FKHR-L1), is a key regulator of lifespan as well as resistance to oxidative or heat stress in response to insulin/insulin-like growth factor signaling (IIS) pathway [46]. And sod-3 is a direct downstream gene of daf-16 [44]. To investigate whether daf-16 could play a role in lifespan extension by HT, we investigated the lifespan of HT acting on daf-16 mutants (GR1307). HT failed to extend the lifespan of daf-16 deletion mutants, indicating that DAF-16 might be a key longevity factor in the HT-dependent longevity mechanism (Figure 6(a)). Considering that the nuclear localization of DAF-16 is the essential prerequisite for DAF-16 transcriptional activation [47], we examined the effect of HT on the nuclear localization of DAF-16 by using DAF-16::GFP transgenic worms TJ356. It was found that HT affected the subcellular distribution of DAF-16 and induced translocation of DAF-16 from the cytoplasm to the nucleus (Figures 6(b) and 6(c)). Specifically, HT treatment induced an increase in nuclear and intermediate DAF-16::GFP translocation, especially in 
nuclear localization from $20 \%$ to $57 \%$ (Figures 6(b) and 6(c)). Meanwhile, the cytosolic DAF-16::GFP ratio was decreased from $46 \%$ to $20 \%$ (Figures 6(b) and 6(c)). Therefore, these results collectively suggested that HTmediated improvement of lifespan and healthspan might be positively linked to the IIS pathway, which might activate the target gene sod-3 by regulating the nuclear localization of DAF-16 in C. elegans.

\section{Conclusion}

In this study, we found that $10 \mathrm{mg} / \mathrm{ml} \mathrm{HT}$ did not produce obvious toxicity to worms, which prolonged lifespan and improved the health of C. elegans without significant damage to physiological function. First, the lifespan was improved by HT without affecting fertility. Meanwhile, the stress resistance and proteotoxicity-induced paralysis were significantly improved. Moreover, HT significantly inhibited the accumulation of lipid and age pigmentation. Finally, we initially elucidated that the HT-mediated health-enhancing effect was positively associated with increased SOD-3 expression and enhanced nuclear translocation of DAF-16 in an IISdependent manner. In conclusion, this work provided the first insight into the health promotion role of HT and elucidates its underlying mechanism. Further studies on dietary HT intake in mouse models or humans are worthy of future research.

\section{Abbreviations}

$\begin{array}{ll}\text { A } \beta \text { : } & \text { Alzheimer } \beta \text {-amyloid } \\ \text { C. elegans: } & \text { Caenorhabditis elegans } \\ \text { CI: } & \text { Chemotaxis index } \\ \text { CK: } & \text { Control check } \\ \text { E. coli OP50: } & \text { Escherichia coli strain OP50 } \\ \text { IIS: } & \begin{array}{l}\text { Insulin/insulin-like growth factor } \\ \text { signaling }\end{array} \\ \text { LC-Q-TOF-MS/MS: } & \text { Liquid chromatography coupled with } \\ & \text { quadrupole time of flight mass } \\ & \text { spectrometry } \\ \text { NGM: } & \text { Nematode growth medium } \\ \text { polyQ: } & \text { Polyglutamine } \\ \text { TIC: } & \text { Total ion chromatograms } \\ \text { HT: } & \text { Herbal tea. }\end{array}$

\section{Data Availability}

The data used to support the findings of this study are included within the article and the supplementary information file.

\section{Ethical Approval}

This article does not contain any studies with human or vertebrate animals.

\section{Conflicts of Interest}

The authors declare that there are no conflicts of interest.

\section{Authors' Contributions}

CL designed and conducted research, analyzed data, performed statistical analysis, and drafted the manuscript. XZ and $\mathrm{CZ}$ conducted research, analyzed data, and performed statistical analysis. YL was responsible for chart making. YC (Yong Cao) conceived the experiment and revised the manuscript. YC (Yunjiao Chen) provided important intellectual contribution, designed and supervised the study, and revised the manuscript. All authors approved the final version of the manuscript.

\section{Acknowledgments}

This research was supported by the National Natural Science Foundation of China (31700501), the Guangdong Innovative and Entrepreneurial Research Team Program (2019ZT08N291), and the Science and Technology Planning Project of Guangzhou (201803010105). We are very grateful to Prof. Qinghua Zhou (Biomedical Translational Research Institute, Jinan University, Guangdong Province, China) for giving the TJ356 transgenic worm and the VC433 and CF1038 mutant strains. The other C. elegans strains were provided by the Caenorhabditis Genetics Center at University of Minnesota.

\section{Supplementary Materials}

Figure S1: representative TIC of HT. Table S1: analysis of various chemical constituents in HT. (Supplementary materials)

\section{References}

[1] T. Finkel and N. J. Holbrook, "Oxidants, oxidative stress and the biology of ageing," Nature, vol. 408, no. 6809, pp. 239$247,2000$.

[2] A. Ghasemzadeh, H. Z. E. Jaafar, and A. Rahmat, "Phytochemical constituents and biological activities of different extracts of Strobilanthes crispus (L.) Bremek leaves grown in different locations of Malaysia," BMC Complementary and Alternative Medicine, vol. 15, no. 1, 2015.

[3] C. G. Pereira, L. Barreira, S. Bijttebier et al., "Health promoting potential of herbal teas and tinctures from Artemisia campestris subsp. maritima: from traditional remedies to prospective products," Scientific Reports, vol. 8, no. 1, p. 4689, 2018.

[4] R. You, Q. Pang, and L. Li, "A metabolic phenotyping approach to characterize the effects of Cantonese herbal tea on restraint stressed rats," Biological \& Pharmaceutical Bulletin, vol. 37, no. 9, pp. 1466-1474, 2014.

[5] R. R. He, B. Tsoi, Y. F. Li, X. S. Yao, and H. Kurihara, "The anti-stress effects of Guangdong herbal tea on immunocompromise in mice loaded with restraint stress," Journal of Health Science, vol. 57, no. 3, pp. 255-263, 2011.

[6] C. Y. Jiang, J. W. Huang, C. Jie et al., "Wanglaoji herbal tea protects against influenza-induced pneumonia in restraintstressed mice via its anti-inflammatory effects," International Journal of Pharmacology, vol. 14, no. 3, pp. 342351, 2018.

[7] H. W. Wei Wang, Y. Zhang, and Y. Zu, "In vitro antioxidant and antimicrobial activity of anthotaxy extracts from 
Dendranthema morifolium (Ramat.) Tzvel. and Chrysanthemum indicum L," Journal of Medicinal Plants Research, vol. 7, no. 36, pp. 2657-2661, 2013.

[8] L. H. Lin, J. H. Xie, S. C. Liu, M. Y. Shen, W. Tang, and M. Y. Xie, "Polysaccharide from Mesona chinensis: extraction optimization, physicochemical characterizations and antioxidant activities," International Journal of Biological Macromolecules, vol. 99, pp. 665-673, 2017.

[9] Y. Pan, L. J. Wu, and Z. L. Yu, "Effect of salt and drought stress on antioxidant enzymes activities and SOD isoenzymes of liquorice (Glycyrrhiza uralensis Fisch)," Plant Growth Regulation, vol. 49, no. 2-3, pp. 157-165, 2006.

[10] G. W. Zhang, L. He, and M. M. Hu, "Optimized ultrasonicassisted extraction of flavonoids from Prunella vulgaris L. and evaluation of antioxidant activities in vitro," Innovative Food Science \& Emerging Technologies, vol. 12, no. 1, pp. 18-25, 2011.

[11] H. T. Wu, G. Zhang, L. S. Huang et al., "Hepatoprotective effect of polyphenol-enriched fraction from Folium Microcos on oxidative stress and apoptosis in acetaminophen-induced liver injury in mice," Oxidative Medicine and Cellular Longevity, vol. 2017, 14 pages, 2017.

[12] O. N. Seo, G. S. Kim, S. Park et al., "Determination of polyphenol components of Lonicera japonica Thunb. using liquid chromatography-tandem mass spectrometry: contribution to the overall antioxidant activity," Food Chemistry, vol. 134, no. 1, pp. 572-577, 2012.

[13] A. J. Merina, D. Sivanesan, V. H. Begum, and N. Sulochana, "Antioxidant and hypolipidemic effect of Plumeria rubra L. in alloxan induced hyperglycemic rats," E-Journal of Chemistry, vol. 7, no. 1, pp. 1-5, 2010.

[14] C. H. Foyer and G. Noctor, "Redox homeostasis and antioxidant signaling: a metabolic interface between stress perception and physiological responses," Plant Cell, vol. 17, no. 7, pp. 1866-1875, 2005.

[15] R. Baumeister and L. M. Ge, "The worm in us - Caenorhabditis elegans as a model of human disease," Trends in Biotechnology, vol. 20, no. 4, pp. 147-148, 2002.

[16] C. Kenyon, "A conserved regulatory system for aging," Cell, vol. 105, no. 2, pp. 165-168, 2001.

[17] L. Guarente and C. Kenyon, "Genetic pathways that regulate ageing in model organisms," Nature, vol. 408, no. 6809, pp. 255-262, 2000.

[18] Y. Chen, B. Onken, H. Chen et al., "Mechanism of longevity extension of Caenorhabditis elegans induced by pentagalloyl glucose isolated from eucalyptus leaves," Journal of Agricultural and Food Chemistry, vol. 62, no. 15, pp. 3422-3431, 2014.

[19] C. Lin, Z. Su, J. Luo et al., "Polysaccharide extracted from the leaves of Cyclocarya paliurus (Batal.) Iljinskaja enhanced stress resistance in Caenorhabditis elegans via skn-1 and hsf-1," International Journal of Biological Macromolecules, vol. 143, pp. 243-254, 2019.

[20] C. D. Link, "C-elegans models of age-associated neurodegenerative diseases: lessons from transgenic worm models of Alzheimer's disease," Experimental Gerontology, vol. 41, no. 10, pp. 1007-1013, 2006.

[21] Y. Wu, Z. Wu, P. Butko et al., "Amyloid-beta-induced pathological behaviors are suppressed by Ginkgo biloba extract EGb 761 and ginkgolides in transgenic Caenorhabditis elegans," The Journal of Neuroscience: The Official Journal of the Society for Neuroscience, vol. 26, no. 50, pp. 13102-13113, 2006.
[22] W. J. Cai, J. H. Huang, S. Q. Zhang et al., "Icariin and its derivative icariside II extend healthspan via insulin/IGF-1 pathway in C. elegans," PLoS One, vol. 6, no. 12, p. e28835, 2011.

[23] Z. Pincus, T. C. Mazer, and F. J. Slack, "Autofluorescence as a measure of senescence in C. elegans: look to red, not blue or green," Aging, vol. 8, no. 5, pp. 889-898, 2016.

[24] C. Lin, Y. Lin, Y. Chen et al., "Effects of Momordica saponin extract on alleviating fat accumulation in Caenorhabditis elegans," Food \& Function, vol. 10, no. 6, pp. 3237-3251, 2019.

[25] C. Lin, X. Zhang, J. Xiao et al., "Effects on longevity extension and mechanism of action of carnosic acid in Caenorhabditis elegans," Food \& Function, vol. 10, no. 3, pp. 1398-1410, 2019.

[26] C. Lin, X. Zhang, Z. Su et al., "Carnosol improved lifespan and healthspan by promoting antioxidant capacity in Caenorhabditis elegans," Oxidative Medicine and Cellular Longevity, vol. 2019, 13 pages, 2019.

[27] H. Li, D. Han, Y. Hou, H. Chen, and Z. Chen, "Statistical inference methods for two crossing survival curves: a comparison of methods," PLoS One, vol. 10, no. 1, article e0116774, 2015.

[28] G. J. Lithgow and G. A. Walker, "Stress resistance as a determinate of C. elegans lifespan," Mechanisms of Ageing and Development, vol. 123, no. 7, pp. 765-771, 2002.

[29] K. Pietsch, N. Saul, S. Chakrabarti, S. R. Sturzenbaum, R. Menzel, and C. E. Steinberg, "Hormetins, antioxidants and prooxidants: defining quercetin-, caffeic acid- and rosmarinic acid-mediated life extension in C. elegans," Biogerontology, vol. 12, no. 4, pp. 329-347, 2011.

[30] R. I. Morimoto, "Stress, aging, and neurodegenerative disease," The New England Journal of Medicine, vol. 355, no. 21, pp. 2254-2255, 2006.

[31] G. McColl, B. R. Roberts, A. P. Gunn et al., "The Caenorhabditis elegans A beta 1-42 model of Alzheimer disease predominantly expresses A beta 3-42," The Journal of Biological Chemistry, vol. 284, no. 34, pp. 22697-22702, 2009.

[32] P. A. Temussi, L. Masino, and A. Pastore, "From Alzheimer to Huntington: why is a structural understanding so difficult?," The EMBO Journal, vol. 22, no. 3, pp. 355-361, 2003.

[33] H. J. Teede, C. Lombard, and A. A. Deeks, "Obesity, metabolic complications and the menopause: an opportunity for prevention," Climacteric, vol. 13, no. 3, pp. 203-209, 2010.

[34] B. Gerstbrein, G. Stamatas, N. Kollias, and M. Driscoll, "In vivo spectrofluorimetry reveals endogenous biomarkers that report healthspan and dietary restriction in Caenorhabditis elegans," Aging Cell, vol. 4, no. 3, pp. 127-137, 2005.

[35] B. Lakowski and S. Hekimi, "The genetics of caloric restriction in Caenorhabditis elegans," Proceedings of the National Academy of Sciences of the United States of America, vol. 95, no. 22, pp. 13091-13096, 1998.

[36] K. Koch, N. Weldle, S. Baier, C. Buchter, and W. Watjen, "Hibiscus sabdariffa L. extract prolongs lifespan and protects against amyloid- $\beta$ toxicity in Caenorhabditis elegans: involvement of the FoxO and Nrf2 orthologues DAF-16 and SKN-1," European Journal of Nutrition, vol. 59, no. 1, pp. 137-150, 2020.

[37] C. Lin, J. Xiao, Y. Xi et al., "Rosmarinic acid improved antioxidant properties and healthspan via the IIS and MAPK pathways in Caenorhabditis elegans," BioFactors, vol. 45, no. 5, pp. 774-787, 2019.

[38] W. Guo, L. Wang, Y. Gao et al., "Isolation of isochlorogenic acid isomers in flower buds of Lonicera japonica by high- 
speed counter-current chromatography and preparative high performance liquid chromatography," Journal of Chromatography $B$, vol. 981, pp. 27-32, 2015.

[39] Z. Cao, Y. Ding, L. Cao, G. Ding, Z. Wang, and W. Xiao, "Isochlorogenic acid C prevents enterovirus 71 infection via modulating redox homeostasis of glutathione," Scientific Reports, vol. 7, no. 1, p. 16278, 2017.

[40] S. M. Tsao and M. C. Yin, "Retraction of "Antioxidative and antiinflammatory activities of asiatic acid, glycyrrhizic acid, and oleanolic acid in human bronchial epithelial cells"," Journal of Agricultural and Food Chemistry, vol. 65, no. 15, pp. 3251-3251, 2017.

[41] C. Guo, S. Wang, J. Duan et al., "Protocatechualdehyde protects against cerebral ischemia-reperfusion-induced oxidative injury via protein kinase $\mathrm{C} \varepsilon / \mathrm{Nrf} 2 / \mathrm{HO}-1$ pathway," Molecular Neurobiology, vol. 54, no. 2, pp. 833-845, 2017.

[42] I. Sadowska-Bartosz and G. Bartosz, "Effect of antioxidants supplementation on aging and longevity," BioMed Research International, vol. 2014, 17 pages, 2014.

[43] P. B. L. Pun, J. Gruber, S. Y. Tang et al., "Ageing in nematodes: do antioxidants extend lifespan in Caenorhabditis elegans?," Biogerontology, vol. 11, no. 1, pp. 17-30, 2010.

[44] Y. Honda and S. Honda, "The daf-2 gene network for longevity regulates oxidative stress resistance and $\mathrm{Mn}$-superoxide dismutase gene expression in Caenorhabditis elegans," The FASEB Journal, vol. 13, no. 11, pp. 1385-1393, 1999.

[45] C. Yee, W. Yang, and S. Hekimi, "The intrinsic apoptosis pathway mediates the pro-longevity response to mitochondrial ROS in C. elegans," Cell, vol. 157, no. 4, pp. 897-909, 2014.

[46] C. T. Murphy, S. A. McCarroll, C. I. Bargmann et al., "Genes that act downstream of DAF-16 to influence the lifespan of Caenorhabditis elegans," Nature, vol. 424, no. 6946, pp. 277283, 2003.

[47] A. Kampkotter, C. G. Nkwonkam, R. F. Zurawski et al., "Effects of the flavonoids kaempferol and fisetin on thermotolerance, oxidative stress and FoxO transcription factor DAF-16 in the model organism Caenorhabditis elegans," Archives of Toxicology, vol. 81, no. 12, pp. 849-858, 2007. 